\title{
ON THE MINIMAL MODELS FOR GRAPH MAPS
}

\author{
LL. ALSEDÀ \\ Departament de Matemàtiques, Universitat Autònoma de Barcelona, \\ 08913 Cerdanyola del Vallès, Barcelona, Spain. \\ On leave at: Departament de Matemàtica Aplicada I, Universitat \\ Politècnica de Catalunya, Diagonal 647, 08028 Barcelona, Spain \\ E-mail: alseda@mat.uab.es \\ D. JUHER \\ Departament d'Informàtica i Matemàtica Aplicada, Universitat de \\ Girona, Lluís Santaló s/n, 17071 Girona, Spain \\ E-mail: juher@ima.udg.es \\ P. MUMBRÚ \\ Departament de Matemàtica Aplicada i Anàlisi, Universitat de Barcelona, \\ Gran Via 585, 08071 Barcelona, Spain \\ E-mail: mumbru@mat.ub.es
}

Received January 2001

The aim of this paper is to present a survey on the existence of minimal models for graph maps and its consequences.

\section{Introduction}

A classical problem in combinatorial dynamics is the following: Given a topological space $X$, a continuous map $f: X \longrightarrow X$ and a finite $f$-invariant set $A \subset X$, what can be said about the dynamics (periodic orbits, topological entropy, ...) of $f$ in terms of $\left.f\right|_{A}$ ? This question can be reworded as follows: What can be said about the dynamics of any continuous map $g: Y \longrightarrow Y$ for which there exists a homeomorphism $\varphi: X \rightarrow Y$ such that $\left.g \circ \varphi\right|_{A}=\left.\varphi \circ f\right|_{A}$ ?

A classical (and well known) case is when $X$ is a closed interval $I$ of the real line. Indeed, if $f: I \longrightarrow I$ is a continuous map then intrinsic information can be obtained by consider- ing the "pattern" of $A$ which is characterized essentially by the permutation $\pi_{A}$ induced by $\left.f\right|_{A}$ (see [Alsedà et al., 2000a] for a precise definition). To each pattern $\pi_{A}$ we may associate a (nonunique) interval map $f_{\pi}$ which admits a finite invariant set $B$, such that the permutation induced by $\left.f_{\pi}\right|_{B}$ is $\pi_{A}$ and $f_{\pi}$ is monotone between consecutive points of $B$. Such a map is called a canonical representative of $\pi_{A}$, or a "connect-the-dots" map. It has the following important properties:

(A) $f_{\pi}$ minimizes topological entropy within the class of interval maps admitting a periodic orbit whose pattern is $\pi_{A}$.

(B) $f_{\pi}$ admits a Markov partition which gives a good "coding" to describe the dynamics of the 
map $f_{\pi}$. The topological entropy of $f_{\pi}$ may be calculated from this partition.

(C) $f_{\pi}$ is essentially unique.

(D) the pattern $\pi_{A}$ forces a pattern $\rho$ if and only if $f_{\pi}$ has a periodic orbit whose pattern is $\rho$. We recall the definition that a pattern $\pi_{A}$ forces a pattern $\pi_{B}$ if and only if each map exhibiting the pattern $\pi_{A}$ also exhibits the pattern $\pi_{B}$ (see [Alsedà et al., 2000a] and [Misiurewicz \& Nitecki, 1991]). In this sense, the dynamics of $f_{\pi}$ are minimal within the class of maps admitting a periodic orbit whose pattern is $\pi_{A}$.

One may make analogies with the study of the surface homeomorphisms where the canonical representatives are given by the Nielsen-Thurston Theorem [Fathi et al., 1979, Thurston, 1988]. In this context the "pattern" or braid type bt $(f, A)$ of a periodic orbit $A$ of a surface homeomorphism $f: M \longrightarrow M$ is characterized by the isotopy class (up to conjugacy) of $\left.f\right|_{M \backslash A}$ [Bowen, 1978, Boyland, 1984, Matsuoka, 1989]. The permutation group arising in the interval case is now replaced by the mapping class group of $M \backslash A$. Analogues of the properties (A)-(D) above are satisfied for each canonical homeomorphism (see [Fathi et al., 1979] for the analogue of properties (A)-(C) and [Asimov \& Franks, 1983, Hall, 1991] for the analogue of (D)). Remarkably, each canonical homeomorphism has an underlying one-dimensional structure, and its dynamics may be calculated from a particular class of continuous graph maps (the fundamental group of the graph is identified with that of the surface) [Bestvina \& Handel, 1995, Los, 1993].

In view of what has been said it is natural to study the above program for the class of continuous graph maps. This is the aim of this paper. We essentially outline the results from the paper [Alsedà et al., 2000b] on the existence and properties of the minimal models for graph maps (Sec. 3), from [Alsedà et al., 1997] for the case of tree maps (Sec. 4) and from [Alsedà et al., 2000c] (Sec. 5). These last results are a consequence of the existence of minimal models for graphs maps. The next section is devoted to introduce the basic notation and definitions that will be used throughout the paper.

\section{Basic Notation and Definitions}

By an interval we mean the closed interval $[0,1]$ and any space homeomorphic to it. A graph is a connected Hausdorff space $G$ which is the union of finitely many intervals $G_{i}$ and $G_{i} \cap G_{j}$ is finite for each $i \neq j$. Note that any graph is compact. A circuit of $G$ is any subset of $G$ homeomorphic to a circle.

Any point of a graph $G$ for which there does not exist an open neighborhood homeomorphic to an open interval of the real line will be called a vertex of $G$. Let $z$ be a vertex of $G$. If $z$ has an open neighborhood homeomorphic to an interval (not necessarily open) of the real line, then it will be called an endpoint of $G$. Otherwise it will be called a branching point of $G$. The set of endpoints and branching points of $G$ will be denoted by $\operatorname{End}(G)$ and $\operatorname{Br}(G)$, respectively.

A tree is a uniquely arcwise-connected graph. Given $A \subset T$ we will denote the convex hull of $A$ (that is, the smallest closed connected set containing $A)$ by $\langle A\rangle_{T}$ or by $\langle A\rangle$.

Any continuous map from a graph (resp. tree) to itself will be called a graph map (resp. tree map).

Next we introduce a notion that will allow us to talk about monotonicity of a map "between" consecutive points of an invariant set for graph maps.

Let $G^{\prime}$ and $G$ be graphs, let $f: G^{\prime} \longrightarrow G$ be a continuous map and let $x \in G^{\prime}$. We denote by $K_{f}(x)$ the (closed) connected component of $f^{-1}(f(x))$ which contains $x$ (notice that if $f$ does not have flat spots then all sets $K_{f}(x)$ are singletons). Let $I$ be a subset of $G^{\prime}$ which is either an interval or a circuit and let $x \in I$. We will say that $\left.f\right|_{I}$ is locally monotone at $x$ if either $K_{\left.f\right|_{I}}(x)=I$ or there exists $V$, an open (in $I$ ) neighborhood of $K_{\left.f\right|_{I}}(x)$, such that $\mathrm{Cl}(V)$ is an interval and $\left.f\right|_{\mathrm{Cl}(V)}$ is monotone (in particular $f(\mathrm{Cl}(V))$ is an interval). We will say that $f$ is monotone on $I$ if it is locally monotone at each point of $I$. Also observe that the path described by $f(x)$ when $x$ ranges over $I$ can wrap several times around a circuit (and thus $\left.f\right|_{I}$ is not injective) but this does not prevent the map $\left.f\right|_{I}$ of being monotone.

The above definition can also be stated as follows. Let $J \subset I$ be homeomorphic to an open interval of the real line. We will say that $\mathrm{Cl}(J)$ is a trivial interval of $I$ if $f(\operatorname{Bd}(J))$ reduces to a point 
and $\left.f\right|_{\mathrm{Cl}(J)}$ is homotopic (relative to $\mathrm{Bd}(J)$ ) to a constant map. Observe that a trivial interval can actually be a circuit. Then, it is easy to see that $f$ is monotone on $I$ if and only if $f$ restricted to any trivial interval of $I$ is constant. If in above definition $G$ and $G^{\prime}$ are trees (and, hence, $I$ is an interval) then $f$ is monotone on $I$ if and only if $f(I)$ is homeomorphic to an interval (perhaps degenerate) and $\left.f\right|_{I}$ is monotone as an interval map.

Let $G$ be a graph, and let $A$ be a finite subset of $G$. The pair $(G, A)$ will be called a pointed graph. Let $f: G \longrightarrow G$ be a graph map and assume that $A$ is $f$-invariant. The triple $(G, A, f)$ will be called a fully specified invariant set. The family of all fully specified invariant sets will be denoted by $\mathcal{F}$.

Let $(G, A, f) \in \mathcal{F}$ and let $I$ be a connected component of $G \backslash(A \cup \operatorname{Br}(G))$ which contains an endpoint of $G$. If we are interested only in the dynamics of $f$ "forced" by $A$ we can remove $I$ from the graph $G$ (and compose $f$ with the retraction to the new graph) without negative consequences for our purposes. This motivates the following notation. Let $(G, A)$ be a pointed graph and let $H$ denote the union of all connected components of $G \backslash(A \cup \operatorname{Br}(G))$ which contain an endpoint of $G$. Then $(G \backslash H, A)$ is still a pointed graph. Clearly, after repeating this procedure finitely many times, we obtain a pointed graph which we will denote by $\left(\langle\langle A\rangle\rangle_{G}, A\right)$ (or simply by $(\langle\langle A\rangle\rangle, A)$ if no confusion is possible) such that $\operatorname{End}(\langle\langle A\rangle\rangle) \subset A$. Observe that the closure of $G \backslash\langle\langle A\rangle\rangle$ consists of finitely many connected components which are trees. Therefore, for each $x \in G \backslash\left\langle\langle A\rangle\right.$ there exists a unique point $z_{x} \in$ $\left\langle\langle A\rangle \cap T_{x}\right.$ where $T_{x}$ is the closure of the connected component of $G \backslash\langle\langle A\rangle\rangle$ that contains $x$. Hence, we can define the natural retraction $\rho: G \longrightarrow\langle\langle A\rangle\rangle$ as

$$
\rho(x)= \begin{cases}x & \text { if } x \in\langle\langle A\rangle, \\ z_{x} & \text { otherwise }\end{cases}
$$

Then, we will denote by $f_{\langle\langle A\rangle}$ the map $\rho \circ f:\langle\langle A\rangle\rangle \longrightarrow\langle\langle A\rangle\rangle$. It is not difficult to see that the maps $\left.f\right|_{\langle A A\rangle}$ and $f_{\langle\langle A\rangle}$ are homotopic relative to $A$. Observe that when $G$ is a tree, then $\langle\langle A\rangle\rangle$ is simply $\langle A\rangle$.

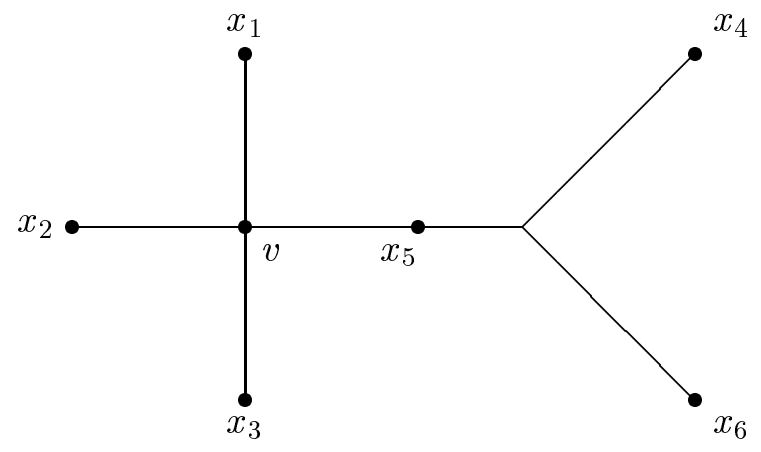

Fig. 1. The cycle of Example 3.1.

\section{Rigid Patterns and Minimal Models on Graphs}

We will start by trying to perform the program described in the introduction for graph maps in a straightforward way. To this end we define an equivalence relation $\widetilde{\mathrm{p}}$ in $\mathcal{F}$ as follows. Given $(G, A, f),\left(G^{\prime}, A^{\prime}, f^{\prime}\right) \in \mathcal{F}$ we write $(G, A, f) \widetilde{\widetilde{\mathrm{p}}}$ $\left(G^{\prime}, A^{\prime}, f^{\prime}\right)$ if and only if there exists a homeomorphism $\phi:\langle\langle A\rangle\rangle_{G} \longrightarrow\left\langle\left\langle A^{\prime}\right\rangle\right\rangle_{G^{\prime}}$ such that $\phi(A)=A^{\prime}$ and the maps $\phi^{-1} \circ f_{\left\langle\left\langle A^{\prime}\right\rangle\right.}^{\prime} \circ \phi$ and $f_{\langle A\rangle\rangle}$ are homotopic relative to $A$. Consequently, $\left.\phi^{-1} \circ f^{\prime} \circ \phi\right|_{A}=\left.f\right|_{A}$. The equivalence classes of fully specified invariant sets in the above relation will be called rigid patterns. As usual the class of $(G, A, f)$ will be denoted by $[G, A, f]$.

A "connect-the-dots" interval map is characterized by the fact that it is monotone between consecutive points of the given invariant set. The first difficulty that one finds in this context is that the "connect-the-dots" map of a given rigid pattern may not exist in this framework. To see it consider the following example:

Example 3.1. Let $(T, A, f)$ be a fully specified invariant set where $T$ is the tree shown in Fig. $1, A=$ $\left\{x_{1}, x_{2}, \ldots, x_{6}\right\}$ and $f\left(x_{i}\right)=x_{i+1}$ for $i=1, \ldots, 5$ and $f\left(x_{6}\right)=x_{1}$. Suppose that we want to construct a tree map $g: T \longrightarrow T$ such that $A$ is $g$-invariant (that is $(T, A, g) \in \mathcal{F}$ ) and $g$ is monotone on each interval of $T$ whose interior does not contain points of $A$. We should have:

$$
\begin{aligned}
& g\left(\left\langle x_{1}, x_{2}\right\rangle\right)=\left\langle x_{2}, x_{3}\right\rangle \\
& g\left(\left\langle x_{3}, x_{5}\right\rangle\right)=\left\langle x_{4}, x_{6}\right\rangle .
\end{aligned}
$$




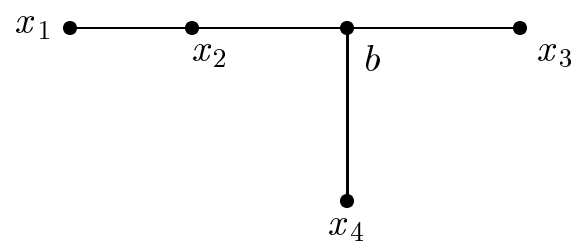

Fig. 2. The tree and maps from Example 3.2.

Thus, we must have $g(v) \in\left\langle x_{2}, x_{3}\right\rangle \cap\left\langle x_{4}, x_{6}\right\rangle$ which is a contradiction.

The consequence of the above example is that, for a rigid pattern, it may happen that there does not exist any representative $(G, A, f)$ such that $f$ is monotone between adjacent points of $A$. One of the possible solutions to this problem is to look for a monotone map between consecutive points of $A \cup \operatorname{Br}(G)$ instead of just consecutive points of $A$. This is the strategy that we adopt in this section while in the next section we will consider another one. This notion can be formalized in the following way. A fully specified invariant set $(G, A, f)$ will be called monotone if

(i) $f(\operatorname{Br}(\langle\langle A\rangle\rangle)) \subset A \cup \operatorname{Br}(\langle\langle A\rangle\rangle)$, and

(ii) $f$ restricted to the closure of every connected component of $\langle\langle A\rangle\rangle \backslash(A \cup \operatorname{Br}(\langle\langle A\rangle\rangle))$ is monotone (in particular $f(\langle\langle A\rangle\rangle) \subset\langle\langle A\rangle\rangle)$ and $f$ is constant on every connected component of $G \backslash\langle\langle A\rangle\rangle$.

Clearly, for each fully specified invariant set there exists a monotone fully specified invariant set with the same rigid pattern. However, the problem is that monotone fully specified invariant sets in general do not satisfy Property (D) above. This is shown, already for tree maps, by the following example:

Example 3.2. Let $T$ be the tree shown in Figure 2, let $A=\left\{x_{1}, x_{2}, x_{3}, x_{4}\right\}$ and let $(T, A, f),(T, A, g) \in$ $\mathcal{F}$ be such that $f\left(x_{i}\right)=g\left(x_{i}\right)=x_{i+1}$ for $i=1,2,3$, $f\left(x_{4}\right)=g\left(x_{4}\right)=x_{1}, f(b)=b$ and $g(b)=x_{1}$ and which are monotone in the closures of the connected components of $T \backslash(A \cup\{b\})$. Clearly, both fully specified invariant sets are monotone and $[T, A, f]=[T, A, g]$ (that is, $(T, A, f)$ and $(T, A, g)$ have the same rigid pattern). Observe that $f$ has a periodic orbit $Q$ of period 3 contained in $\left\langle x_{1}, x_{2}\right\rangle \cup\left\langle b, x_{3}\right\rangle \cup\left\langle b, x_{4}\right\rangle$ while each periodic orbit of period 3 of $g$ is contained in $\left\langle x_{1}, x_{3}\right\rangle$. These periodic orbits of $f$ and $g$ live in spaces not homeomorphic, so they have different rigid patterns. Therefore we have found two "connect-the-dots" models of the same rigid pattern such that each of them has rigid patterns that the other fully specified invariant set does not have. Hence, Property (D) above does not hold for this notion of "connect-the-dots" model.

The above example suggests that, given a rigid pattern $\mathcal{P}$, there does not exist a monotone fully specified invariant set with rigid pattern $\mathcal{P}$ having a minimal set of rigid patterns forced by $\mathcal{P}$. Another natural question is: Does there exist a monotone fully specified invariant set that minimizes the topological entropy within the class of all representatives of a given rigid pattern? In the rest of this section we will show that indeed there exist monotone fully specified invariant sets with a given rigid pattern that minimize the entropy of this rigid pattern. To state this result we need some more notation. First let us define what we understand by minimizing the entropy of a rigid pattern.

Given a rigid pattern $\mathcal{P}$ we define the entropy of $\mathcal{P}$ by

$$
\inf \{h(f):(G, A, f) \in \mathcal{P}\}
$$

and we denote it by $h(\mathcal{P})$.

Let $(G, A, f) \in \mathcal{F}$ be monotone. A point $v \in \operatorname{Br}(\langle\langle A\rangle\rangle)$ will be called free if $K_{f}(v) \cap A=\emptyset$ (in particular $v \notin A$ ). Moreover, we will say that $(G, A, f)$ is direct at $v$ if for every neighborhood $U$ of $K_{f}(v)$ it follows that $f(v) \in \operatorname{Int}(f(U))$ (with the topology of $f(U))$. When $(G, A, f)$ is monotone and direct at each free branching point we will say that $(G, A, f)$ is a direct fully specified invariant set.

Theorem A. Given a rigid pattern $\mathcal{P}=[G, A, f]$ there exists a direct representative $\left(\langle A A\rangle_{G}, A, g\right) \in \mathcal{P}$ such that $h(g)=h(\mathcal{P})$.

Notice that the above result is an existence theorem and, in principle, it does not provide an algorithm to construct the direct representative of the rigid pattern which gives its entropy. However, for tree maps this is not the case. Indeed, let $[T, A, f]$ be a rigid pattern where $T$ is a tree. 
Notice that $\langle\langle A\rangle\rangle=\langle A\rangle$ and $(\langle A\rangle, A, g)$ is a representative of $[T, A, f]$ if and only if $\left.g\right|_{A}=\left.f\right|_{A}$. Moreover, if $(\langle A\rangle, A, g)$ is a monotone representative of $[T, A, f]$ then the transition matrix of the $g$-graph of $A \cup \operatorname{Br}(\langle A\rangle)$ is uniquely determined by the choice of $g$ on $\operatorname{Br}(\langle A\rangle) \backslash A$. Since there are finitely many possibilities for $\left.g\right|_{\operatorname{Br}(\langle A\rangle) \backslash A}$ in order that $(\langle A\rangle, A, g)$ be monotone, the set of transition matrices of all direct representatives of $[T, A, f]$ is finite. Thus, the above theorem gives, in practice, a finite algorithm to compute $h([T, A, f])$.

\section{Patterns and Minimal Models on Trees}

In the previous section we have seen that, given a rigid pattern $\mathcal{P}$, it may happen that there does not exist any representative of $\mathcal{P}$ which is monotone between consecutive points of $A$ (see Example 3.1). In this section we will adopt the strategy of maintaining the combinatorial structure of the points of the invariant set (and, in particular, their relative positions) while releasing the rigidity of the space. This will give rise to a new notion of pattern for which there will exist monotone models in the above sense. This notion will fulfill the above program (that is, Properties (A-D)). Unfortunately this theory, up to now, only exists for tree maps.

Let $(T, A)$ be a pointed tree. A nonempty set $Q \subset A$ is said to be a discrete component of $(T, A)$ if either $|Q|>1$ and there exists a connected component $C$ of $T \backslash A$ such that $Q=A \cap \mathrm{Cl}(C)$, or $|Q|=1$ and $Q=A$. If $Q$ and $Q^{\prime}$ are two distinct discrete components of $(T, A)$ then $Q \cap Q^{\prime}$ is either empty or contains a unique element. Any (unordered) binary subset of a discrete component will be called a basic path of $(T, A)$.

We now introduce an equivalence relation on pointed trees. We say that two pointed trees $(T, A)$ and $\left(T^{\prime}, A^{\prime}\right)$ are equivalent, written $(T, A) \sim$ $\left(T^{\prime}, A^{\prime}\right)$, if there exists a bijection $\phi: A \longrightarrow A^{\prime}$ which preserves discrete components (and, therefore, their relative positions). Thus $Q$ is a discrete component of $(T, A)$ if and only if $\phi(Q)$ is a discrete component of $\left(T^{\prime}, A^{\prime}\right)$. The equivalence class of a pointed tree $(T, A)$ will be denoted by $[T, A]$. Observe that with this definition, the topologies of $T$ and $T^{\prime}$ may be different. A class of pointed trees is determined solely by the discrete components and their relative positions.
Let $(T, A)$ and $\left(T^{\prime}, A^{\prime}\right)$ be equivalent pointed trees, and let $\theta: A \longrightarrow A$ and $\theta^{\prime}: A^{\prime} \longrightarrow A^{\prime}$ be maps. We will say that $\theta$ and $\theta^{\prime}$ are equivalent, written $\theta \approx \theta^{\prime}$, if and only if $\theta^{\prime}=\phi \circ \theta \circ \phi^{-1}$ for a bijection $\phi: A \longrightarrow A^{\prime}$ which preserves discrete components. The equivalence class of $\theta$ by the relation $\approx$ will be denoted by $[\theta]$. If $\mathcal{T}=[T, A]$ is an equivalence class of pointed trees and if $\Theta=[\theta]$ is an equivalence class of maps then the pair $(\mathcal{T}, \Theta)$ will be called a pattern.

A fully specified invariant set $(T, A, f)$ is said to have the pattern $\left([T, A],\left[\left.f\right|_{A}\right]\right)$. Also, a tree map $f: T \longrightarrow T$ is said to exhibit the pattern $(\mathcal{T}, \Theta)$ if there exists $(T, A, f) \in \mathcal{F}$ having pattern $(\mathcal{T}, \Theta)$.

To each pattern $(\mathcal{T}, \Theta)=([T, A],[\theta])$, we will associate a path transition matrix as follows. Let $\left\{\pi_{1}, \pi_{2}, \ldots, \pi_{k}\right\}$ be the set of basic paths of $(T, A)$. The $k \times k$ matrix $M_{P}(\mathcal{T}, \Theta)=\left(m_{i j}\right)$ defined by

$$
m_{i j}= \begin{cases}1 & \text { if } \pi_{j} \subset\left\langle\theta\left(\pi_{i}\right)\right\rangle_{T} \\ 0 & \text { otherwise }\end{cases}
$$

will be called the path transition matrix of $(\mathcal{T}, \Theta)$. From above it follows that this definition is independent of the particular choice of $T, A$ and $\theta$. Thus the path transition matrix is a well-defined pattern invariant.

Now let $(T, A, f)$ be a fully specified invariant set. We say that $(T, A, f)$ is $A$-monotone if $f$ is constant on each connected component of $T \backslash\langle A\rangle$ and, for each basic path $\pi$ of $(T, A)$ it follows that $f(\langle\pi\rangle)=\langle f(\pi)\rangle$ and $\left.f\right|_{\langle\pi\rangle}$ is monotone. It can be shown that, in such a case, the image of each vertex is uniquely determined and is either a vertex or belongs to $A$. Thus actually the $A$-monotone fully specified invariant sets are monotone but not conversely.

The entropy of a pattern $(\mathcal{T}, \Theta)$, denoted by $h(\mathcal{T}, \Theta)$, is defined to be the infimum of the entropies of all tree maps which exhibit the pattern $(\mathcal{T}, \Theta)$.

Let $M$ be a square matrix. We shall denote by $\rho(M)$ the spectral radius of $M$. The main result of this section is the following.

Theorem B. Let $(\mathcal{T}, \Theta)$ be a pattern. Then the following statements hold.

(a) There exists an A-monotone fully specified invariant set $(T, A, f)$ having pattern $(\mathcal{T}, \Theta)$. 
(b) $h(f)=h(\mathcal{T}, \Theta)$ for each A-monotone fully specified invariant set $(T, A, f)$ which has pattern $(\mathcal{T}, \Theta)$.

(c) $h(\mathcal{T}, \Theta)=\log \max \left\{\rho\left(M_{P}(\mathcal{T}, \Theta)\right), 1\right\}$.

The above theorem tells us that Properties $(\mathrm{A}, \mathrm{B})$ of the program described above hold for patterns of tree maps. In [Alsedà et al., 1997] it is shown that the $A$-monotone fully specified invariant sets are, in some sense, equivalent and also that, with an appropriate notion of combinatorial type, minimize the dynamics of the pattern. Thus, Properties (C,D) also hold.

\section{An Application of the Minimal Models of Rigid Patterns}

For interval maps it is possible to show that the entropy of any map $f$ is the supremum of the values $h\left(f_{\pi}\right)$ corresponding to all patterns $\pi$ of periodic orbits of $f$. Furthermore, for each $n$, we can take this supremum only over the patterns of period $k>n$. This result was stated by Takahashi [Takahashi, 1980] and proved with the assumption that $f$ is piecewise monotone. In the general case it also was proved in an independent way by Block and Coven [Block \& Coven, 1989] and Misiurewicz and Nitecki [Misiurewicz \& Nitecki, 1991].

The following theorem shows that an analogous relation between periodic behavior and topological entropy is satisfied for continuous maps on graphs.

Theorem C. Let $G$ be a graph and let $f: G \longrightarrow G$ be a graph map. Then, for each nonnegative integer $m$ we have that the topological entropy of $f$ is the supremum of the entropies $h([G, A, f])$ among all periodic orbits $A$ of $f$ such that $|A|>m$.

The entropies $h([G, A, f])$ are well defined and computable thanks to Theorem A.

\section{Acknowledgements}

The authors have been partially supported by the DGES grant number PB96-1153.

\section{References}

Alsedà, Ll., Guaschi, J., Los, J., Mañosas, F. \& Mumbrú, P. [1997] "Canonical representatives for patterns of tree maps," Topology 36, 11231153.

Alsedà, Ll., Llibre, J. \& Misiurewicz, M. [2000] Combinatorial Dynamics and Entropy in Dimension One, Advances Series in Nonlinear Dynamics, Vol. 5, World Scientific, Singapore.

Alsedà, Ll., Mañosas, F. \& Mumbrú, P. [2000] "Minimizing topological entropy for continuous maps on graphs," Ergod. Th. \& Dynam. Sys. 20, 1559-1576.

Alsedà, Ll., Juher, D. \& Mumbrú, P. [2000] “A note on the periodic orbits and topological entropy of graph maps," to appear in Proc. Amer. Math. Soc.

Asimov, D. \& Franks, J. [1983] "Unremovable closed orbits," Lecture Notes in Mathematics, Vol. 1007, Springer-Verlag, Berlin, pp. 22-29.

Bestvina, M. \& Handel, M. [1995] "Train tracks for surface homeomorphisms," Topology 34, 109140.

Block, L. S. \& Coven, E. [1989] "Approximating entropy of maps of the interval," in Proceedings of the semester on Ergodic Theory and Dynamical Systems, Banach Center Pub., Vol. 23, PWN, Warsaw, pp. 237-241.

Bowen, R. [1978] "Entropy and the fundamental group," Lecture Notes in Mathematics, Vol. 668, Springer-Verlag, Berlin, pp. 21-29.

Boyland, P. L. [1984] "Braid types and a topological method of proving positive entropy," preprint.

Denker, M., Grillenberger, C. \& Sigmund, K. [1976] "Ergodic Theory on Compact Spaces," Lecture Notes in Mathematics, Vol. 527, SpringerVerlag, Berlin.

Fathi, A., Laudenbach, F. \& Poénaru, V. [1979] Travaux de Thurston sur les surfaces, Astérisque 66-67. 
Hall, T. [1991] "Unremovable periodic orbits of homeomorphisms," Math. Proc. Cambridge Phil. Soc. 110, 523-531.

Los, J. [1993] "Pseudo-Anosov maps and invariant train tracks in the disc: a finite algorithm," Proc. London Math. Soc. 66, 400-430.

Matsuoka, T. [1983] "The number and linking of periodic solutions of periodic systems," Invent. Math. 20, 319-340.

Misiurewicz, M. \& Nitecki, Z. [1991] "Combinatorial patterns for maps of the interval," Mem. Amer. Math. Soc. 94.

Takahashi, Y. [1980] "A formula for topological entropy of one-dimensional dynamics," Sci. Papers College Gen. Ed. Univ. Tokyo 30, 11-22.

Thurston, J. P. [1988] "On the geometry and dynamics of surfaces," Bull. Amer. Math. Soc. 19, 417-431. 